\title{
Nurses Intent to Leave and Job Satisfaction in Hematology/Oncology Areas: Implications for Policy and Practice
}

\author{
Mohammad Suhail Dagamseh RN, BSN, MSc ${ }^{*}$ and Ahlam Haddad RN, BSN, CPHQ
}

King Faisal Specialist Hospital and Research Center, Nursing Education, Altakhasossi, Riyadh, Saudi Arabia

*Corresponding author: Mohammad Suhail Dagamseh, Education Coordinator, King Faisal Specialist Hospital and Research Center, Nursing Education, Altakhasossi, Riyadh, 11211, Saudi Arabia, Tel; 00966506457270; E-mail: mdagamseh@kfshrc.edu.sa

Received date: March 02, 2016; Accepted date: August 31, 2016; Published date: September 08, 2016

Copyright: (c) 2016 Dagamseh MS, et al. This is an open-access article distributed under the terms of the Creative Commons Attribution License, which permits unrestricted use, distribution, and reproduction in any medium, provided the original author and source are credited

\begin{abstract}
Purpose/objective: To determine how satisfied the nurses are in the Hematology/Oncology areas, to find what extent the expatriate nurses intend to leave their jobs, and to find the relationship between job satisfaction and turnover intention in Hematology/Oncology units. The focus of this study was on measuring the effect of the demographic characteristics of the participants on their intent to leave and to link those characteristics to their job satisfaction.
\end{abstract}

Design: Quantitative descriptive cross sectional research.

Method: Study instruments included questions related to demographic characteristics, intent to leave, and the McCloskey Mueller Satisfaction Scale. Data were extracted and analyzed using Statistical Package for Social Scientists (20.V). Univariate descriptive statistics were conducted on the sample's demographic characteristics including gender, age, marital status, nationality and educational level. Bivariate associations between intent to leave and demographic characteristics were tested using Pearson Chi-square. Differences in satisfaction scores between nurses with and without intent to leave were tested using t-test and ANOVA f-test.

Results: A total of 223 (68.6\% response rate) expatriate nurses employed in the Hematology/Oncology units were surveyed.104 (46.6\%) of the participants reported intent to leave within the next 1-3 years. Leavers were less satisfied in all satisfaction subscales than the stayers. Significant Differences were found between marital status and nationalities on one side and intention to leave on the other side ( $P$ value $=0.027,0.014$, respectively). Predictors of intent to leave were dissatisfaction with different job aspects.

Implications for nursing: Findings can be utilized by nursing managers and policy makers to enhance job satisfaction and use it as predictor for intent to leave.

Conclusion: Study results predict relationships between job satisfaction and intent to leave the employment within Hematology/Oncology areas or the organization.

Keywords: Expatriate; Hematology/oncology; Intent to leave; Job satisfaction; Nurses; Nursing turnover

\section{Background}

Turnover among nurses is a significant phenomenon that persists in health care organizations [1-8]. It is a major issue both in the developed and the developing countries [9]. The shortage in the latter mentioned countries mostly happens as a result of nurses' emigration from middle and low income countries to the industrial countries [9].

There is no doubt about the effect of turnover on the job satisfaction and retention of workers including nurses [10]. The higher the turnover rates the lower the retention reached and the more adverse influence on the patients' outcome. Turnover negatively impacts the patient care as it is the leading cause of the shortage in "qualified and skilled nurses", at the same time; it adds more cost to the process of selection and training of the newly hired nurses [5].
The traditional strategies taken by health care organizations focused on recruitment in order to compensate shortage in nurses that might result from the turnover [11]. However, these strategies have failed to alleviate the shortage of nurses. This necessitates the focus on retention strategies to keep nurses in their places [11-13].

Nursing turnover is connected negatively to the retention. High turnover leads to an increased burden of work, lack of nurse to patient interaction, lower opportunities for development, and lower level of nurses' morale [14]. In addition to that, turnover leads to higher rate of accidents and absenteeism among remaining nurses [15,16]. Likewise, nursing turnover impacts safe medication practice by increasing errors, decreasing the channels of communication among nurses and between nurses and other healthcare providers which results in poor patient satisfaction $[17,18]$.

The Kingdom of Saudi Arabia (KSA) has a chronic shortage of Saudi nurses accompanied by high rate of turnover [19]. Therefore, the expatriate nurses form more than $70 \%$ of the nursing work force in the Kingdom of Saudi Arabia [20]. The demand for both native and 
expatriate nurses will increase in the upcoming years as the Saudi population is growing and health care facilities are expanding [20]. This dependence on the expatriate's nurses in KSA to satisfy its national nursing demand makes studying the job satisfaction and intent to leave amongst those nurses who make up the vast majority of nursing manpower in KSA an important area to study [15]. Up to this nursing era, researchers have significantly neglected this population of nurses who become expatriates outside their countries of origin [15]. Therefore, the available literature fails to explain the reasons behind the turnover among expatriate nurses who are working outside their countries. On the other hand, few researchers have explained it by struggling to adapt with the new culture and the new way of life at working and social levels [15,21].

\section{Design and Sample}

A quantitative descriptive cross sectional research design was utilized to survey expatriate nurses currently working in one of the largest tertiary hospitals in Riyadh-KSA in the Hematology/Oncology $(\mathrm{H} / \mathrm{O})$ units in order to find to which extent those nurses are planning to leave and what is the relationship between job satisfaction and their intent to leave. The focus of this study was on measuring the effect of the demographic characteristics of the participants on their intent to leave and to link those characteristics to their job satisfaction.

The study sample included all expatriate registered nurses who provide direct care to cancer patients and who are employed fulltime in the $\mathrm{H} / \mathrm{O}$ units where the study has taken place. All participants in the study were registered nurses in KSA and were working in the organization for more than ninety days. Nine H/O units were included in the study. Each unit was given a letter-name in the study instrument which was the same name of the unit used in the hospital with the specialty description of each unit (e.g. B3: Pediatric Hematology and stem cell transplantation). A total of 325 nurses from 35 different nationalities met the criteria and were included in the study. Native nurses were excluded from the study as they do not meet the employment criteria of the study.

\section{Instruments}

The questionnaire consisted of three sections. The first section was on demographic data of the nurses. The second section which was established by El-Jardali et al. [3,4] in 2009 included questions about intent to leave and plans after leaving. This section was modified to meet elements characteristics and to better fit the context of the study. Leaving the unit was defined as transfer to another unit or leaving the entire hospital without specification. Furthermore, McCloskey-Mueller Satisfaction Scale (MMSS) (Table 1) was used to assess job satisfaction of the participants. The original scale consisted of 31 items on a 5-point Likert scale ranging from 1 (very dissatisfied) to 5 (very satisfied). Question number 20 in the original MMSS questionnaire was removed as it does not apply to the hospital setting. The order of all questions from number 20 was changed after excluding this question from the tool. The questionnaires together with the cover letter were provided in English language and distributed to the participants. Nurses were given one month to fill the questionnaires and returned them back to the assigned locked box.

\begin{tabular}{|c|c|c|c|c|c|c|}
\hline & & $\begin{array}{l}\text { Very } \\
\text { Dissatisfied }\end{array}$ & Dissatisfied & Neutral & Satisfied & $\begin{array}{l}\text { Very } \\
\text { Satisfied }\end{array}$ \\
\hline 1 & Salary & 1 & 2 & 3 & 4 & 5 \\
\hline 2 & Vacation & 1 & 2 & 3 & 4 & 5 \\
\hline 3 & Benefit package (insurance, retirement) & 1 & 2 & 3 & 4 & 5 \\
\hline 4 & Hours that you work & 1 & 2 & 3 & 4 & 5 \\
\hline 5 & Flexibility in scheduling your hours & 1 & 2 & 3 & 4 & 5 \\
\hline 6 & $\begin{array}{l}\text { Opportunity to work straight days (answer this question as Neutral if you are } \\
\text { working in an outpatient area) }\end{array}$ & 1 & 2 & 3 & 4 & 5 \\
\hline 7 & Opportunity for part-time work & 1 & 2 & 3 & 4 & 5 \\
\hline 8 & Weekends off per month & 1 & 2 & 3 & 4 & 5 \\
\hline 9 & $\begin{array}{l}\text { Flexibility in scheduling your weekends (answer this question as Neutral if you } \\
\text { are working in an outpatient area) }\end{array}$ & 1 & 2 & 3 & 4 & 5 \\
\hline 10 & $\begin{array}{l}\text { Compensation for working weekends (answer this question as Neutral if you } \\
\text { are working in an outpatient area) }\end{array}$ & 1 & 2 & 3 & 4 & 5 \\
\hline 11 & Maternity leave time & 1 & 2 & 3 & 4 & 5 \\
\hline 12 & Child care facilities for employees' children in the hospital & 1 & 2 & 3 & 4 & 5 \\
\hline 13 & Your head nurse & 1 & 2 & 3 & 4 & 5 \\
\hline 14 & Your nursing peers & 1 & 2 & 3 & 4 & 5 \\
\hline 15 & The physicians you work with & 1 & 2 & 3 & 4 & 5 \\
\hline
\end{tabular}


Citation: Dagamseh MS, Haddad A (2017) Nurses Intent to Leave and Job Satisfaction in Hematology/Oncology Areas: Implications for Policy and Practice. J Nurs Care 5: 363. doi:10.4172/2167-1168.1000363

Page 3 of 15

\begin{tabular}{|c|c|c|c|c|c|c|}
\hline 16 & $\begin{array}{l}\text { The delivery of care method used on your unit (e.g., functional, team, primary, } \\
\text { modular care, patient-centered care) }\end{array}$ & 1 & 2 & 3 & 4 & 5 \\
\hline 17 & Opportunities for social contact at work & 1 & 2 & 3 & 4 & 5 \\
\hline 18 & Opportunities for social contact with your colleagues after work & 1 & 2 & 3 & 4 & 5 \\
\hline 19 & Opportunities to interact professionally with other disciplines & 1 & 2 & 3 & 4 & 5 \\
\hline 20 & Opportunities to belong to department and institutional committees & 1 & 2 & 3 & 4 & \\
\hline 21 & Control over what goes on in your work setting & 1 & 2 & 3 & 4 & 5 \\
\hline 22 & Opportunities for career advancement & 1 & 2 & 3 & 4 & 5 \\
\hline 23 & Recognition of your work from superiors & 1 & 2 & 3 & 4 & 5 \\
\hline 24 & Recognition of your work from peers & 1 & 2 & 3 & 4 & 5 \\
\hline 25 & Amount of encouragement and positive feedback & 1 & 2 & 3 & 4 & 5 \\
\hline 26 & Opportunities to participate in nursing research & 1 & 2 & 3 & 4 & 5 \\
\hline 27 & Opportunities to write and publish & 1 & 2 & 3 & 4 & 5 \\
\hline 28 & Your amount of responsibility & 1 & 2 & 3 & 4 & 5 \\
\hline 29 & Your control of work conditions & 1 & 2 & 3 & 4 & 5 \\
\hline 30 & Your participation in organizational decision making & 1 & 2 & 3 & 4 & 5 \\
\hline
\end{tabular}

Table 1: McCloskey-Mueller Satisfaction Scale (MMSS) McCloskey Mueller Satisfaction Scale (MMSS) (๔ 1989), How satisfied are you with the following aspects of your current job?

\section{Data Analysis}

The Statistical Package of Social Sciences (SPSS), Version 20, was used for all analysis. Univariate descriptive statistic tests were computed to summarize the demographic characteristics. Chi- square test was used to find any differences between intent to leave and demographic characteristics, while a t-test and one-way variance of analysis (ANOVA) were used. At another stage, correlation coefficient tests (Pearson's) was used to find the relationship and effects between grand job satisfaction (summation of all subscales) and each subscale.

\section{Findings}

A total of 223 questionnaires were fully completed with a response rate of (68.6\%). Majority were females $84.8 \%$, Asian nationalities $54.3 \%$, age between 30 and 45 years $49.3 \%$, ever married $58.7 \%$ and holding a Bachelor Degree $74 \%$ (Table 2).

\begin{tabular}{|l|l|l|l|}
\hline Characteristic & & $\mathbf{n}$ & $\mathbf{\%}$ \\
\hline \multirow{4}{*}{ Gender } & Female & 189 & $84.80 \%$ \\
\hline \multirow{4}{*}{ Age, years } & Male & 34 & $15.20 \%$ \\
\hline Marital Status & $<30$ & 77 & $34.50 \%$ \\
\cline { 2 - 5 } & $30-45$ & 110 & $49.30 \%$ \\
\cline { 2 - 5 } & $46-55$ & 24 & $10.80 \%$ \\
\cline { 2 - 5 } & $>55$ & 12 & $5.40 \%$ \\
\hline
\end{tabular}

\begin{tabular}{|c|c|c|c|}
\hline & Ever Married & 131 & $58.70 \%$ \\
\hline \multirow[b]{4}{*}{ Educational degree } & Diploma & 52 & $23.30 \%$ \\
\hline & Bachelor & 165 & $74.00 \%$ \\
\hline & Master & 3 & $1.30 \%$ \\
\hline & Other & 3 & $1.30 \%$ \\
\hline \multirow[b]{9}{*}{ Working unit } & B3 & 38 & $17.00 \%$ \\
\hline & CWA & 22 & $9.90 \%$ \\
\hline & CWB & 13 & $5.80 \%$ \\
\hline & CCC-OPD & 21 & $9.40 \%$ \\
\hline & Cancer Center-OPD & 38 & $17.00 \%$ \\
\hline & $\mathrm{E} 1$ & 21 & $9.40 \%$ \\
\hline & E3 & 17 & $7.60 \%$ \\
\hline & $\mathrm{F} 2-2$ & 26 & $11.70 \%$ \\
\hline & $\mathrm{F} 3$ & 27 & $12.10 \%$ \\
\hline \multirow[b]{5}{*}{ Nationality } & Africa & 12 & $5.40 \%$ \\
\hline & Arab & 51 & $22.90 \%$ \\
\hline & Asia & 121 & $54.30 \%$ \\
\hline & Australia & 7 & $3.10 \%$ \\
\hline & Europe & 14 & $6.30 \%$ \\
\hline
\end{tabular}


Citation: Dagamseh MS, Haddad A (2017) Nurses Intent to Leave and Job Satisfaction in Hematology/Oncology Areas: Implications for Policy and Practice. J Nurs Care 5: 363. doi:10.4172/2167-1168.1000363

Page 4 of 15

\begin{tabular}{|l|l|l|l|}
\hline & North America & 6 & $2.70 \%$ \\
\cline { 2 - 4 } & others & 12 & $5.40 \%$ \\
\hline
\end{tabular}

leaving, $58 \%$ of the leavers were planning to leave the hospital or the country, (21\%) planning to leave working at $\mathrm{H} / \mathrm{O}$ areas, and $(6 \%)$ planning to leave the nursing profession. (50\%) thought it is easy to find another job in nursing and $26 \%$ thought it is difficult. (24\%) were not sure. A majority (70\%) reported that they would choose nursing as

Table 2: Demographic characteristics of sampled nurses. a profession if they were given the chance again to start all over, and

\section{Intent to leave and plans} (30\%) reported not to select nursing profession again (Table 3).

The participants reported close intention to leave (46.6\% Leavers) to intention to stay (53.4\% Stayers). When asked about their plans after

\begin{tabular}{|c|c|c|c|c|c|c|c|}
\hline & & \multicolumn{4}{|c|}{ Thinking about the next $1-3$ years } & & \multirow[b]{3}{*}{ P-Value } \\
\hline & & \multicolumn{2}{|c|}{ Intend to stay } & \multicolumn{2}{|c|}{ Intend to leave } & \multirow[b]{2}{*}{ Test } & \\
\hline & & $\mathbf{n}$ & $\%$ & $\mathbf{n}$ & $\%$ & & \\
\hline \multirow{2}{*}{ Gender } & Female & 96 & $50.80 \%$ & 93 & $49.20 \%$ & Chi-Square & 0.07 \\
\hline & Male & 23 & $67.60 \%$ & 11 & $32.40 \%$ & & \\
\hline \multirow{4}{*}{ Age, y } & $<30$ & 43 & $55.80 \%$ & 34 & $44.20 \%$ & Chi-Square & 0.832 \\
\hline & $30-45$ & 58 & $52.70 \%$ & 52 & $47.30 \%$ & & \\
\hline & $46-55$ & 13 & $54.20 \%$ & 11 & $45.80 \%$ & & \\
\hline & $>55$ & 5 & $41.70 \%$ & 7 & $58.30 \%$ & & \\
\hline \multirow[b]{2}{*}{ Marital Status } & Never Married & 41 & $44.60 \%$ & 51 & $55.40 \%$ & Chi-Square & 0.027 \\
\hline & Ever Married & 78 & $59.50 \%$ & 53 & $40.50 \%$ & & \\
\hline \multirow{4}{*}{ Educational degree } & Diploma & 31 & $59.60 \%$ & 21 & $40.40 \%$ & Cramer's V & 0.628 \\
\hline & Bachelor & 85 & $51.50 \%$ & 80 & $48.50 \%$ & & \\
\hline & Master & 2 & $66.70 \%$ & 1 & $33.30 \%$ & & \\
\hline & Other & 1 & $33.30 \%$ & 2 & $66.70 \%$ & & \\
\hline \multirow{9}{*}{ Working unit } & B3 & 18 & $47.40 \%$ & 20 & $52.60 \%$ & Chi-Square & 0.583 \\
\hline & CWA & 12 & $54.50 \%$ & 10 & $45.50 \%$ & & \\
\hline & CWB & 7 & $53.80 \%$ & 6 & $46.20 \%$ & & \\
\hline & CCC-OPD & 13 & $61.90 \%$ & 8 & $38.10 \%$ & & \\
\hline & Cancer Centre-OPD & 23 & $60.50 \%$ & 15 & $39.50 \%$ & & \\
\hline & E1 & 11 & $52.40 \%$ & 10 & $47.60 \%$ & & \\
\hline & E3 & 6 & $35.30 \%$ & 11 & $64.70 \%$ & & \\
\hline & F2-2 & 17 & $65.40 \%$ & 9 & $34.60 \%$ & & \\
\hline & F3 & 12 & $44.40 \%$ & 15 & $55.60 \%$ & & \\
\hline \multirow{5}{*}{ Nationality } & Africa & 3 & $25.00 \%$ & 9 & $75.00 \%$ & Cramer's V & 0.014 \\
\hline & Arab & 32 & $62.70 \%$ & 19 & $37.30 \%$ & & \\
\hline & Asia & 71 & $58.70 \%$ & 50 & $41.30 \%$ & & \\
\hline & Australia & 1 & $14.30 \%$ & 6 & $85.70 \%$ & & \\
\hline & Europe & 6 & $42.90 \%$ & 8 & $57.10 \%$ & & \\
\hline
\end{tabular}


Citation: Dagamseh MS, Haddad A (2017) Nurses Intent to Leave and Job Satisfaction in Hematology/Oncology Areas: Implications for Policy and Practice. J Nurs Care 5: 363. doi:10.4172/2167-1168.1000363

Page 5 of 15

\begin{tabular}{|c|c|c|c|c|c|c|c|}
\hline & North America & 3 & $50.00 \%$ & 3 & $50.00 \%$ & & \\
\hline & others & 3 & $25.00 \%$ & 9 & $75.00 \%$ & & \\
\hline \multirow{8}{*}{ Plan after leaving current job } & Move to a non- $\mathrm{H} / \mathrm{O}$ Unit & 0 & $0.00 \%$ & 22 & $100.00 \%$ & Cramer's V & 0 \\
\hline & Leave the Hospital & 0 & $0.00 \%$ & 30 & $100.00 \%$ & & \\
\hline & Leave the country & 0 & $0.00 \%$ & 30 & $100.00 \%$ & & \\
\hline & Change nursing profession & 0 & $0.00 \%$ & 6 & $100.00 \%$ & & \\
\hline & Continue education & 0 & $0.00 \%$ & 8 & $100.00 \%$ & & \\
\hline & $\begin{array}{l}\text { Take care of children or } \\
\text { other dependents }\end{array}$ & 0 & $0.00 \%$ & 3 & $100.00 \%$ & & \\
\hline & Other & 0 & $0.00 \%$ & 5 & $100.00 \%$ & & \\
\hline & NA & 119 & $100.00 \%$ & 0 & $0.00 \%$ & & \\
\hline \multirow{3}{*}{$\begin{array}{l}\text { In your opinion, finding another job } \\
\text { in nursing would be }\end{array}$} & Easy & 52 & $46.80 \%$ & 59 & $53.20 \%$ & Chi-Square & 0.104 \\
\hline & Difficult & 37 & $63.80 \%$ & 21 & $36.20 \%$ & & \\
\hline & not sure & 30 & $55.60 \%$ & 24 & $44.40 \%$ & & \\
\hline \multirow{3}{*}{$\begin{array}{l}\text { Given the opportunity to start all } \\
\text { over, would you choose nursing as } \\
\text { a profession }\end{array}$} & Yes & 85 & $54.50 \%$ & 71 & $45.50 \%$ & Chi-Square & 0.489 \\
\hline & No & 24 & $47.10 \%$ & 27 & $52.90 \%$ & & \\
\hline & Not Sure & 10 & $62.50 \%$ & 6 & $37.50 \%$ & & \\
\hline
\end{tabular}

Table 3: Relationship between demographic characteristics and plan to leave or to stay.

\section{Gender}

With respect to gender, those who intend to leave are more likely to be females ( $49.2 \%$ of the total female participants).

\section{Age}

Those who intend to leave are more likely to be more than 50 years old (58\%).

\section{Marital status}

Nurses who planned to leave the hospital or the units are more likely to be never married (55.4\%). In contrast, those who were ever married intend to stay in the hospital or the unit (59.5\%, P-value, 0.027).

\section{Level of education}

$48.5 \%$ of the bachelor holding participants intends to leave.

\section{McCloskey Mueller Satisfaction Scale (MMSS)}

After the analysis of the MMSS scale it was found that leavers, whether leaving the unit or the hospital, were consistently less satisfied in all subscales. Additionally, the analysis revealed that both stayers and leavers were least satisfied with balance between work and family (mean, 2.455) but they were most satisfied with co-workers (3.60) (Figure 1).

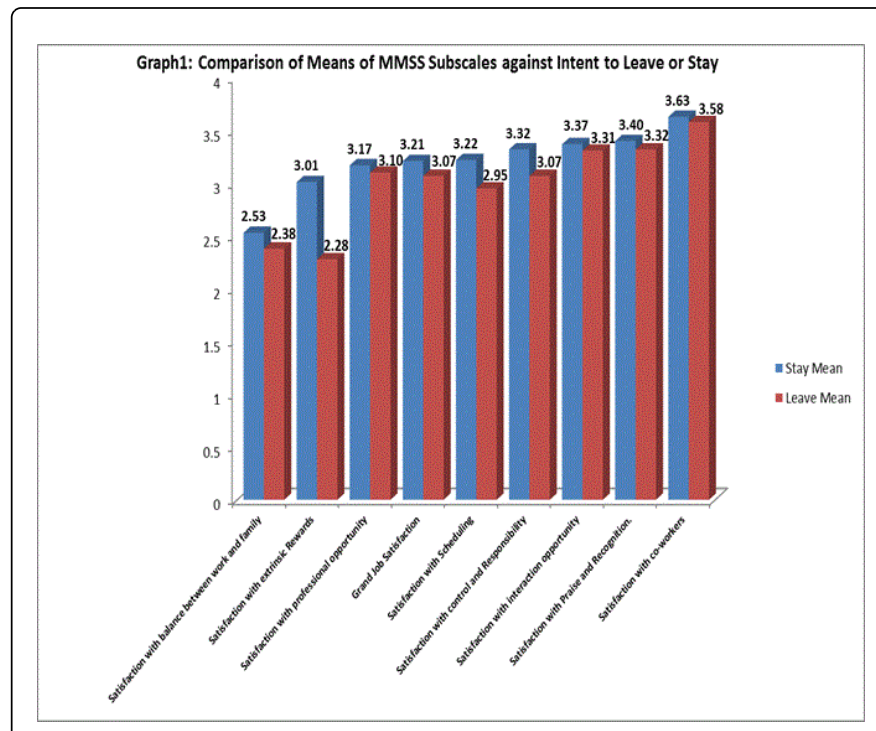

Figure 1: Comparison of means of MMSS subscales against intent to leave or stay.

Differences between sample demographic characteristics and job satisfaction were done applying t-test and ANOVA to find any association with the satisfaction with subscales (Tables $4 \mathrm{~A}-4 \mathrm{~F}$ ). 
Citation: Dagamseh MS, Haddad A (2017) Nurses Intent to Leave and Job Satisfaction in Hematology/Oncology Areas: Implications for Policy and Practice. J Nurs Care 5: 363. doi:10.4172/2167-1168.1000363

Page 6 of 15

\begin{tabular}{|c|c|c|c|c|c|}
\hline & Gender & $\mathbf{n}$ & Mean & SD & $P$ Value \\
\hline \multirow[b]{2}{*}{ Total Nurse Perceived extrinsic Rewards } & Female & 189 & 3 & 0.737 & \multirow[b]{2}{*}{$>0.05$} \\
\hline & Male & 34 & 2.96 & 0.676 & \\
\hline \multirow[b]{2}{*}{ Total Nurse Perceived satisfaction with balance between work and family } & Female & 189 & 2.49 & 0.703 & \multirow[b]{2}{*}{$>0.05$} \\
\hline & Male & 34 & 2.26 & 0.76 & \\
\hline \multirow[b]{2}{*}{ Total Nurse Perceived satisfaction with co-workers } & Female & 189 & 3.61 & 0.578 & \multirow[b]{2}{*}{$>0.05$} \\
\hline & Male & 34 & 3.57 & 0.664 & \\
\hline \multirow[b]{2}{*}{ Total Nurse Perceived satisfaction with interaction opportunity } & Female & 189 & 3.38 & 0.524 & \multirow[b]{2}{*}{0.003} \\
\hline & Male & 34 & 3.16 & 0.593 & \\
\hline \multirow[b]{2}{*}{ Total Nurse Perceived satisfaction with professional opportunity } & Female & 189 & 3.14 & 0.566 & \multirow[b]{2}{*}{$>0.05$} \\
\hline & Male & 34 & 3.14 & 0.575 & \\
\hline \multirow[b]{2}{*}{ Total Nurse Perceived satisfaction Praise and Recognition. } & Female & 189 & 3.39 & 0.575 & \multirow[b]{2}{*}{$>0.05$} \\
\hline & Male & 34 & 3.23 & 0.827 & \\
\hline \multirow[b]{2}{*}{ Total Nurse Perceived satisfaction with control and Responsibility } & Female & 189 & 3.19 & 0.556 & \multirow[b]{2}{*}{$>0.05$} \\
\hline & Male & 34 & 3.27 & 0.595 & \\
\hline \multirow[b]{2}{*}{ Total Nurse satisfaction with Scheduling } & Female & 189 & 3.09 & 0.54 & \multirow[b]{2}{*}{$>0.05$} \\
\hline & Male & 34 & 3.14 & 0.817 & \\
\hline \multirow[b]{2}{*}{ Grand Job Satisfaction } & Female & 189 & 3.16 & 0.387 & \multirow[b]{2}{*}{$>0.05$} \\
\hline & Male & 34 & 3.1 & 0.483 & \\
\hline
\end{tabular}

Table 4A: Gender and satisfaction with MMSS subscales.

\begin{tabular}{|c|c|c|c|c|c|c|c|c|c|}
\hline & \multicolumn{8}{|l|}{ Age } & \multirow{3}{*}{$P$ Value } \\
\hline & \multicolumn{2}{|l|}{$<30$} & \multicolumn{2}{|l|}{$30-45$} & \multicolumn{2}{|l|}{ 46-55 } & \multicolumn{2}{|l|}{$>55$} & \\
\hline & Mean & $\mathbf{n}$ & Mean & $\mathbf{n}$ & Mean & $\mathbf{n}$ & Mean & $\mathbf{n}$ & \\
\hline \multirow[b]{2}{*}{ Total Nurse Perceived extrinsic Rewards } & 3 & 77 & 3 & 11 & 3 & 24 & 3 & 12 & \multirow[b]{2}{*}{$>0.05$} \\
\hline & & & & 0 & & & & & \\
\hline \multirow[b]{2}{*}{ Total Nurse Perceived satisfaction with balance between work and family } & & & & 11 & & & & & \multirow[b]{2}{*}{$>0.05$} \\
\hline & 3 & 77 & 2 & 0 & 2 & 24 & 3 & 12 & \\
\hline \multirow[b]{2}{*}{ Total Nurse Perceived satisfaction with co- workers } & & & & 11 & & & & & \multirow[b]{2}{*}{0.01} \\
\hline & 4 & 77 & 4 & 0 & 3 & 24 & 3 & 12 & \\
\hline \multirow[b]{2}{*}{ Total Nurse Perceived satisfaction with interaction opportunity } & & & & 11 & & & & & \multirow[b]{2}{*}{0.02} \\
\hline & 3 & 77 & 3 & 0 & 3 & 24 & 3 & 12 & \\
\hline \multirow[b]{2}{*}{ Total Nurse Perceived satisfaction with professional opportunity } & & & & 11 & & & & & \multirow[b]{2}{*}{$>0.05$} \\
\hline & 3 & 77 & 3 & 0 & 3 & 24 & 3 & 12 & \\
\hline \multirow[b]{2}{*}{ Total Nurse Perceived satisfaction Praise and Recognition. } & & & & 11 & & & & & \multirow[b]{2}{*}{$>0.05$} \\
\hline & 3 & 77 & 3 & 0 & 3 & 24 & 3 & 12 & \\
\hline
\end{tabular}


Citation: Dagamseh MS, Haddad A (2017) Nurses Intent to Leave and Job Satisfaction in Hematology/Oncology Areas: Implications for Policy and Practice. J Nurs Care 5: 363. doi:10.4172/2167-1168.1000363

Page 7 of 15

\begin{tabular}{|c|c|c|c|c|c|c|c|c|c|}
\hline & & & & 11 & & & & & \\
\hline Total Nurse Perceived satisfaction with control and Responsibility & 3 & 77 & 3 & 0 & 3 & 24 & 3 & 12 & $>0.05$ \\
\hline \multirow[b]{2}{*}{ Total Nurse satisfaction with Scheduling } & 3 & 77 & 3 & 11 & 3 & 24 & 3 & 12 & \multirow[b]{2}{*}{$>0.05$} \\
\hline & & & & 0 & & & & & \\
\hline \multirow[b]{2}{*}{ Grand Job Satisfaction } & 3 & 77 & 3 & 11 & 3 & 24 & 3 & 12 & \multirow[b]{2}{*}{$>0.05$} \\
\hline & & & & 0 & & & & & \\
\hline
\end{tabular}

Table 4B: Age and satisfaction with MMSS subscales.

\begin{tabular}{|c|c|c|c|c|c|}
\hline & Marital Status & $\mathbf{n}$ & Mean & SD & P Value \\
\hline \multirow[b]{2}{*}{ Total Nurse Perceived extrinsic Rewards } & Never Married & 92 & 3.1 & 0.702 & $>0.05$ \\
\hline & Ever Married & 131 & 2.93 & 0.738 & \\
\hline \multirow[b]{2}{*}{ Total Nurse Perceived satisfaction with balance between work and family } & Never Married & 92 & 2.64 & 0.61 & 0,001 \\
\hline & Ever Married & 131 & 2.33 & 0.757 & \\
\hline \multirow[b]{2}{*}{ Total Nurse Perceived satisfaction with co- workers } & Never Married & 92 & 3.65 & 0.582 & $>0.05$ \\
\hline & Ever Married & 131 & 3.58 & 0.596 & \\
\hline \multirow[b]{2}{*}{ Total Nurse Perceived satisfaction with interaction opportunity } & Never Married & 92 & 3.35 & 0.534 & $>0.05$ \\
\hline & Ever Married & 131 & 3.34 & 0.544 & \\
\hline \multirow[b]{2}{*}{ Total Nurse Perceived satisfaction with professional opportunity } & Never Married & 92 & 3.14 & 0.53 & $>0.05$ \\
\hline & Ever Married & 131 & 3.13 & 0.592 & \\
\hline \multirow[b]{2}{*}{ Total Nurse Perceived satisfaction Praise and Recognition. } & Never Married & 92 & 3.43 & 0.555 & $>0.05$ \\
\hline & Ever Married & 131 & 3.32 & 0.661 & \\
\hline \multirow[b]{2}{*}{ Total Nurse Perceived satisfaction with control and Responsibility } & Never Married & 92 & 3.19 & 0.571 & $>0.05$ \\
\hline & Ever Married & 131 & 3.21 & 0.557 & \\
\hline \multirow[b]{2}{*}{ Total Nurse satisfaction with Scheduling } & Never Married & 92 & 3.03 & 0.552 & $>0.05$ \\
\hline & Ever Married & 131 & 3.15 & 0.61 & \\
\hline \multirow[b]{2}{*}{ Grand Job Satisfaction } & Never Married & 92 & 3.17 & 0.379 & $>0.05$ \\
\hline & Ever Married & 131 & 3.13 & 0.419 & \\
\hline
\end{tabular}

Table 4C: Marital status and satisfaction with MMSS subscales.

\begin{tabular}{|c|c|c|c|c|c|c|c|c|c|}
\hline & \multicolumn{8}{|c|}{ What is your highest degree } & \multirow{3}{*}{ P Value } \\
\hline & \multicolumn{2}{|c|}{ Diploma } & \multicolumn{2}{|c|}{ Bachelor } & \multicolumn{2}{|c|}{ Master } & \multicolumn{2}{|l|}{ Other } & \\
\hline & Mean & $\mathbf{n}$ & Mean & $\mathbf{n}$ & Mean & $\mathbf{n}$ & Mean & $\mathbf{n}$ & \\
\hline \multirow[b]{2}{*}{ Grand Job Satisfaction } & 3 & 52 & 3 & 16 & 3 & 3 & 3 & 3 & \multirow[b]{2}{*}{$>0.05$} \\
\hline & & & & 5 & & & & & \\
\hline \multirow[b]{2}{*}{ Total Nurse Perceived extrinsic Rewards } & 3 & 52 & 3 & 16 & 3 & 3 & 3 & 3 & \multirow[b]{2}{*}{$>0.05$} \\
\hline & & & & 5 & & & & & \\
\hline Total Nurse Perceived satisfaction with balance between work and family & & & & 16 & & & & & $>0.05$ \\
\hline
\end{tabular}


Citation: Dagamseh MS, Haddad A (2017) Nurses Intent to Leave and Job Satisfaction in Hematology/Oncology Areas: Implications for Policy and Practice. J Nurs Care 5: 363. doi:10.4172/2167-1168.1000363

Page 8 of 15

\begin{tabular}{|c|c|c|c|c|c|c|c|c|c|}
\hline & 3 & 52 & 2 & 5 & 3 & 3 & 2 & 3 & \\
\hline \multirow[b]{2}{*}{ Total Nurse Perceived satisfaction with co- workers } & & & & 16 & & & & & \multirow[b]{2}{*}{$>0.05$} \\
\hline & 3 & 52 & 4 & 5 & 4 & 3 & 3 & 3 & \\
\hline \multirow[b]{2}{*}{ Total Nurse Perceived satisfaction with interaction opportunity } & & & & 16 & & & & & \multirow[b]{2}{*}{$>0.05$} \\
\hline & 3 & 52 & 3 & 5 & 4 & 3 & 4 & 3 & \\
\hline \multirow[b]{2}{*}{ Total Nurse Perceived satisfaction with professional opportunity } & & & & 16 & & & & & \multirow[b]{2}{*}{$>0.05$} \\
\hline & 3 & 52 & 3 & 5 & 4 & 3 & 3 & 3 & \\
\hline \multirow[b]{2}{*}{ Total Nurse Perceived satisfaction Praise and Recognition. } & & & & 16 & & & & & \multirow[b]{2}{*}{$>0.05$} \\
\hline & 3 & 52 & 3 & 5 & 3 & 3 & 4 & 3 & \\
\hline \multirow[b]{2}{*}{ Total Nurse Perceived satisfaction with control and Responsibility } & & & & 16 & & & & & \multirow[b]{2}{*}{$>0.05$} \\
\hline & 3 & 52 & 3 & 5 & 3 & 3 & 3 & 3 & \\
\hline \multirow[b]{2}{*}{ Total Nurse satisfaction with Scheduling } & 3 & 52 & 3 & 16 & 3 & 3 & 3 & 3 & \multirow[b]{2}{*}{$>0.05$} \\
\hline & & & & 5 & & & & & \\
\hline
\end{tabular}

Table 4D: Level of education and satisfaction with MMSS subscales.

\begin{tabular}{|c|c|c|c|c|c|c|c|c|c|c|c|c|c|c|c|c|c|c|c|}
\hline & \multicolumn{18}{|c|}{ At which unit from the following are you working } & \multirow{3}{*}{$\begin{array}{l}\mathbf{P} \\
\text { Value }\end{array}$} \\
\hline & \multicolumn{2}{|l|}{ B3 } & \multicolumn{2}{|l|}{ CWA } & \multicolumn{2}{|l|}{ CWB } & \multicolumn{2}{|c|}{ CCC-OPD } & \multicolumn{2}{|c|}{$\begin{array}{l}\text { Cancer } \\
\text { Center- } \\
\text { OPD }\end{array}$} & \multicolumn{2}{|l|}{ E1 } & \multicolumn{2}{|l|}{ E3 } & \multicolumn{2}{|l|}{ F2-2 } & \multicolumn{2}{|l|}{ F3 } & \\
\hline & Mean & $\mathbf{n}$ & Mean & $\mathbf{n}$ & Mean & $\mathbf{n}$ & Mean & n & Mean & $\mathbf{n}$ & Mean & $\mathbf{n}$ & Mean & $\mathbf{n}$ & Mean & $\mathbf{n}$ & Mean & n & \\
\hline $\begin{array}{l}\text { Total Nurse Perceived extrinsic } \\
\text { Rewards }\end{array}$ & 3 & 38 & 3 & 22 & 3 & 13 & 3 & 21 & 3 & 38 & 3 & 21 & 3 & 17 & 3 & 26 & 3 & 27 & $>0.05$ \\
\hline $\begin{array}{l}\text { Total Nurse Perceived satisfaction } \\
\text { with balance between work and } \\
\text { family }\end{array}$ & 2 & 38 & 3 & 22 & 2 & 13 & 3 & 21 & 2 & 38 & 2 & 21 & 2 & 17 & 3 & 26 & 2 & 27 & 0 \\
\hline $\begin{array}{l}\text { Total Nurse Perceived satisfaction } \\
\text { with co-workers }\end{array}$ & 4 & 38 & 4 & 22 & 4 & 13 & 4 & 21 & 4 & 38 & 4 & 21 & 3 & 17 & 4 & 26 & 4 & 27 & $>0.05$ \\
\hline $\begin{array}{l}\text { Total Nurse Perceived satisfaction } \\
\text { with interaction opportunity }\end{array}$ & 3 & 38 & 3 & 22 & 3 & 13 & 3 & 21 & 3 & 38 & 3 & 21 & 3 & 17 & 3 & 26 & 3 & 27 & $>0.05$ \\
\hline $\begin{array}{l}\text { Total Nurse Perceived satisfaction } \\
\text { with professional opportunity }\end{array}$ & 3 & 38 & 3 & 22 & 3 & 13 & 3 & 21 & 3 & 38 & 3 & 21 & 3 & 17 & 3 & 26 & 3 & 27 & $>0.05$ \\
\hline $\begin{array}{l}\text { Total Nurse Perceived satisfaction } \\
\text { Praise and Recognition. }\end{array}$ & 3 & 38 & 3 & 22 & 3 & 13 & 3 & 21 & 3 & 38 & 4 & 21 & 4 & 17 & 3 & 26 & 4 & 27 & $>0.05$ \\
\hline $\begin{array}{l}\text { Total Nurse Perceived satisfaction } \\
\text { with control and Responsibility }\end{array}$ & 3 & 38 & 3 & 22 & 3 & 13 & 3 & 21 & 3 & 38 & 3 & 21 & 3 & 17 & 3 & 26 & 3 & 27 & $>0.05$ \\
\hline $\begin{array}{l}\text { Total Nurse satisfaction with } \\
\text { Scheduling }\end{array}$ & 3 & 38 & 3 & 22 & 3 & 13 & 3 & 21 & 3 & 38 & 3 & 21 & 3 & 17 & 3 & 26 & 3 & 27 & $>0.05$ \\
\hline Grand Job Satisfaction & 3 & 38 & 3 & 22 & 3 & 13 & 3 & 21 & 3 & 38 & 3 & 21 & 3 & 17 & 3 & 26 & 3 & 27 & 0.02 \\
\hline
\end{tabular}

Table 4E: Working unit and satisfaction with MMSS subscales. 
Citation: Dagamseh MS, Haddad A (2017) Nurses Intent to Leave and Job Satisfaction in Hematology/Oncology Areas: Implications for Policy and Practice. J Nurs Care 5: 363. doi:10.4172/2167-1168.1000363

Page 9 of 15

\begin{tabular}{|c|c|c|c|c|c|c|c|c|c|c|c|c|c|c|c|}
\hline & \multicolumn{14}{|c|}{ Nationality } & \multirow{3}{*}{ P Value } \\
\hline & \multicolumn{2}{|l|}{ Africa } & \multicolumn{2}{|l|}{ Arab } & \multicolumn{2}{|l|}{ Asia } & \multicolumn{2}{|c|}{ Australia } & \multicolumn{2}{|c|}{ Europe } & \multicolumn{2}{|c|}{$\begin{array}{l}\text { North } \\
\text { America }\end{array}$} & \multicolumn{2}{|c|}{ Others } & \\
\hline & Mean & $\mathbf{n}$ & Mean & $\mathbf{n}$ & Mean & $\mathbf{n}$ & Mean & $\mathbf{n}$ & Mean & $\mathbf{n}$ & Mean & $\mathbf{n}$ & Mean & $\mathbf{n}$ & \\
\hline $\begin{array}{l}\text { Total Nurse Perceived extrinsic } \\
\text { Rewards }\end{array}$ & 3 & 12 & 3 & 51 & 3 & 121 & 3 & 7 & 3 & 14 & & & & & \\
\hline $\begin{array}{l}\text { Total Nurse Perceived } \\
\text { satisfaction with balance } \\
\text { between work and family }\end{array}$ & 2 & 12 & 2 & 51 & 3 & 121 & 2 & 7 & 3 & 14 & & & & & \\
\hline $\begin{array}{l}\text { Total Nurse Perceived } \\
\text { satisfaction with co-workers }\end{array}$ & 3 & 12 & 4 & 51 & 4 & 121 & 3 & 7 & 3 & 14 & & & & & \\
\hline $\begin{array}{lll}\text { Total Nurse } & \text { Perceived } \\
\text { satisfaction with } & \text { interaction } \\
\text { opportunity } & & \end{array}$ & 3 & 12 & 3 & 51 & 3 & 121 & 3 & 7 & 3 & 14 & & & & & \\
\hline 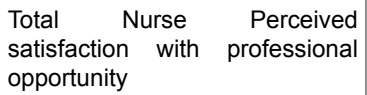 & 3 & 12 & 3 & 51 & 3 & 121 & 3 & 7 & 3 & 14 & & & & & \\
\hline $\begin{array}{l}\text { Total Nurse Perceived } \\
\text { satisfaction Praise and } \\
\text { Recognition. }\end{array}$ & 3 & 12 & 3 & 51 & 3 & 121 & 3 & 7 & 3 & 14 & & & & & \\
\hline $\begin{array}{l}\text { Total Nurse } \\
\text { serceived } \\
\text { satisfaction with control and } \\
\text { Responsibility }\end{array}$ & 3 & 12 & 3 & 51 & 3 & 121 & 3 & 7 & 3 & 14 & & & & & \\
\hline $\begin{array}{l}\text { Total Nurse satisfaction with } \\
\text { Scheduling }\end{array}$ & 3 & 12 & 3 & 51 & 3 & 121 & 3 & 7 & 3 & 14 & & & & & \\
\hline Grand Job Satisfaction & 3 & 12 & 3 & 51 & 3 & 121 & 3 & 7 & 3 & 14 & & & & & \\
\hline
\end{tabular}

Table 4F: Nationalities and satisfaction with MMSS subscales.

Relationships and effects between grand job satisfaction (summation of all subscales) and each subscale were explored using correlation coefficient tests (Pearson's) (Table 5).

\begin{tabular}{|c|c|c|c|c|c|c|c|c|c|c|}
\hline & & $\begin{array}{l}\text { Total } \\
\text { Nurse } \\
\text { Perceive } \\
\text { d } \\
\text { extrinsic } \\
\text { Rewards }\end{array}$ & $\begin{array}{l}\text { Total Nurse } \\
\text { Perceived } \\
\text { satisfaction } \\
\text { with balance } \\
\text { between } \\
\text { work and } \\
\text { family }\end{array}$ & $\begin{array}{l}\text { Total } \\
\text { Nurse } \\
\text { Perceived } \\
\text { satisfactio } \\
\text { n with co- } \\
\text { workers }\end{array}$ & $\begin{array}{l}\text { Total Nurse } \\
\text { Perceived } \\
\text { satisfaction } \\
\text { with } \\
\text { interaction } \\
\text { opportunity }\end{array}$ & $\begin{array}{l}\text { Total Nurse } \\
\text { Perceived } \\
\text { satisfaction } \\
\text { with } \\
\text { professiona } \\
\text { I } \\
\text { opportunity }\end{array}$ & $\begin{array}{l}\text { Total Nurse } \\
\text { Perceived } \\
\text { satisfaction } \\
\text { Praise and } \\
\text { Recognitio } \\
\text { n. }\end{array}$ & $\begin{array}{l}\text { Total Nurse } \\
\text { Perceived } \\
\text { satisfaction } \\
\text { with control } \\
\text { and } \\
\text { Responsibilit } \\
\text { y }\end{array}$ & $\begin{array}{l}\text { Total } \\
\text { Nurse } \\
\text { satisfactio } \\
\text { n with } \\
\text { Schedulin } \\
\text { g }\end{array}$ & $\begin{array}{l}\text { Grand Job } \\
\text { Satisfaction }\end{array}$ \\
\hline \multirow{3}{*}{$\begin{array}{lr}\text { Total } & \text { Nurse } \\
\text { Perceived } & \text { extrinsic } \\
\text { Rewards } & \end{array}$} & $r$ & 1 & $0.315^{\star *}$ & 0.069 & $0.289^{\star *}$ & $0.341^{* *}$ & $0.302^{* *}$ & $0.376^{* *}$ & $0.413^{* *}$ & $0.613^{* *}$ \\
\hline & $\begin{array}{l}\mathrm{P} \\
\text { value }\end{array}$ & 0 & 0.305 & 0 & 0 & 0 & 0 & 0 & 0 & 0 \\
\hline & $n$ & 223 & 223 & 223 & 223 & 223 & 223 & 223 & 223 & 223 \\
\hline \multirow{3}{*}{$\begin{array}{l}\text { Total Nurse } \\
\text { Perceived } \\
\text { satisfaction with } \\
\text { balance between } \\
\text { work and family }\end{array}$} & $r$ & $0.315^{\star *}$ & 1 & 0.011 & 0.111 & $0.262^{\star *}$ & $0.177^{\star *}$ & $0.215^{\star \star}$ & $0.312^{\star *}$ & $0.470^{* *}$ \\
\hline & $\begin{array}{l}\mathrm{P} \\
\text { value }\end{array}$ & 0 & & 0.871 & 0.098 & 0 & 0.008 & 0.001 & 0 & 0 \\
\hline & $n$ & 223 & 223 & 223 & 223 & 223 & 223 & 223 & 223 & 223 \\
\hline \multirow{2}{*}{$\begin{array}{l}\text { Total Nurse } \\
\text { Perceived } \\
\text { satisfaction with co- } \\
\text { workers }\end{array}$} & $r$ & 0.069 & 0.011 & 1 & $0.460^{\star *}$ & $0.208^{\star *}$ & $0.270^{* *}$ & $0.293^{* *}$ & $0.220^{\star \star}$ & $0.412^{* *}$ \\
\hline & $\begin{array}{l}P \\
\text { value }\end{array}$ & 0.305 & 0.871 & & 0 & 0.002 & 0 & & 0.1 & 0 \\
\hline
\end{tabular}


Citation: Dagamseh MS, Haddad A (2017) Nurses Intent to Leave and Job Satisfaction in Hematology/Oncology Areas: Implications for Policy and Practice. J Nurs Care 5: 363. doi:10.4172/2167-1168.1000363

Page 10 of 15

\begin{tabular}{|c|c|c|c|c|c|c|c|c|c|c|}
\hline & $\mathrm{n}$ & 223 & 223 & 223 & 223 & 223 & 223 & 223 & 223 & 223 \\
\hline \multirow{3}{*}{$\begin{array}{l}\text { Total } \\
\text { Perceived } \\
\text { satisfaction } \\
\text { interaction } \\
\text { opportunity }\end{array}$} & $r$ & $0.289^{* *}$ & 0.111 & $0.460^{* *}$ & 1 & $0.480^{* *}$ & $0.453^{* *}$ & $0.497^{\star *}$ & $0.321^{* *}$ & $0.666^{* *}$ \\
\hline & $\begin{array}{l}P \\
\text { value }\end{array}$ & 0 & 0.098 & 0 & & 0 & 0 & 0 & 0 & 0 \\
\hline & $\mathrm{n}$ & 223 & 223 & 223 & 223 & 223 & 223 & 223 & 223 & 223 \\
\hline \multirow{3}{*}{$\begin{array}{l}\text { Total } \\
\text { Perceived } \\
\text { satisfaction } \\
\text { professional } \\
\text { opportunity }\end{array}$} & $r$ & $0.341^{* *}$ & $0.262^{* *}$ & $0.208^{* *}$ & $0.480^{\star *}$ & 1 & $0.456^{* *}$ & $0.606^{* *}$ & $0.339^{* *}$ & $0.689^{* *}$ \\
\hline & $\begin{array}{l}P \\
\text { value }\end{array}$ & 0 & 0 & 0.002 & 0 & & 0 & 0 & 0 & 0 \\
\hline & $\mathrm{n}$ & 223 & 223 & 223 & 223 & 223 & 223 & 223 & 223 & 223 \\
\hline \multirow{3}{*}{$\begin{array}{l}\text { Total } \\
\text { Perceived } \\
\text { satisfaction } \\
\text { and Recogniti }\end{array}$} & $r$ & $0.302^{* *}$ & $0.177^{* *}$ & $0.270^{* *}$ & $0.453^{\star *}$ & $0.456^{* *}$ & 1 & $0.608^{* *}$ & $0.375^{\star *}$ & $0.714^{* *}$ \\
\hline & $\begin{array}{l}P \\
\text { value }\end{array}$ & 0 & 0.008 & 0 & 0 & 0 & & 0 & 0 & 0 \\
\hline & $\mathrm{n}$ & 223 & 223 & 223 & 223 & 223 & 223 & 223 & 223 & 223 \\
\hline \multirow{3}{*}{$\begin{array}{l}\text { Total } \\
\text { Perceived } \\
\text { satisfaction } \\
\text { control } \\
\text { Responsibility }\end{array}$} & $r$ & $0.376^{\star *}$ & $0.215^{\star \star}$ & $0.293^{\star *}$ & $0.497^{\star *}$ & $0.606^{\star *}$ & $0.608^{* *}$ & 1 & $0.473^{* *}$ & $0.805^{\star *}$ \\
\hline & $\begin{array}{l}P \\
\text { value }\end{array}$ & 0 & 0.001 & 0 & 0 & 0 & 0 & & 0 & 0 \\
\hline & $\mathrm{n}$ & 223 & 223 & 223 & 223 & 223 & 223 & 223 & 223 & 223 \\
\hline \multirow{3}{*}{$\begin{array}{l}\text { Total } \\
\text { satisfaction } \\
\text { Scheduling }\end{array}$} & $r$ & $0.413^{* *}$ & $0.312^{* *}$ & $0.220^{* *}$ & $0.321^{* *}$ & $0.339^{* *}$ & $0.375^{\star *}$ & $0.473^{* *}$ & 1 & $0.736^{* *}$ \\
\hline & $\begin{array}{l}P \\
\text { value }\end{array}$ & 0 & 0 & 0.001 & 0 & 0 & 0 & 0 & & 0 \\
\hline & $n$ & 223 & & 223 & 223 & 223 & 223 & 223 & 223 & 223 \\
\hline \multirow{3}{*}{$\begin{array}{l}\text { Grand } \\
\text { Satisfaction }\end{array}$} & $r$ & $0.613^{* *}$ & $0.470^{* *}$ & $0.412^{* *}$ & $0.666^{* \star}$ & $0.689^{* *}$ & $0.714^{* *}$ & $0.805^{* *}$ & $0.736^{* *}$ & 1 \\
\hline & $\begin{array}{l}P \\
\text { value }\end{array}$ & 0 & 0 & 0 & 0 & 0 & 0 & 0 & 0 & \\
\hline & $\mathrm{n}$ & 223 & 223 & 223 & 223 & 223 & 223 & 223 & 223 & 223 \\
\hline
\end{tabular}

Table 5: Correlations between grand job satisfaction and satisfaction subscales.

Correlations of Grand Job Satisfaction and Intention to Leave

To find if there is any relationship between grand job satisfaction and intention to leave, a correlation coefficient (Pearson's) test was conducted. The grand job satisfaction was significantly and inversely associated with leave intent ( $r=-0.179, \mathrm{P}$-value, 0.007) (Table 6).

\begin{tabular}{|c|c|c|c|}
\hline & & Thinking about the next $1-3$ years, do & $\begin{array}{l}\text { Grand } \\
\text { Satisfaction }\end{array}$ \\
\hline \multirow[t]{3}{*}{ Thinking about the next $1-3$ years, do you } & Pearson Correlation & 1 & $-0.179^{* *}$ \\
\hline & Sig. (2-tailed) & & 0.007 \\
\hline & $\mathrm{N}$ & 223 & 223 \\
\hline \multirow[t]{3}{*}{ Grand Job Satisfaction } & Pearson Correlation & $-0.179^{\star \star}$ & 1 \\
\hline & Sig. (2-tailed) & & 0.007 \\
\hline & $\mathrm{N}$ & 223 & 223 \\
\hline
\end{tabular}


Citation: Dagamseh MS, Haddad A (2017) Nurses Intent to Leave and Job Satisfaction in Hematology/Oncology Areas: Implications for Policy and Practice. J Nurs Care 5: 363. doi:10.4172/2167-1168.1000363

Page 11 of 15

** Correlation is significant at the 0.01 level (2-tailed)

Table 6: Correlations of grand job satisfaction and intention to leave.

\section{Plans after leaving current job}

Participants were rearranged into two main groups, those who wanted to leave the unit or those who wanted to leave the hospital. The analysis has shown a significant relationship between having a plan after leaving current job and intent to leave (P-value, 0.000). In like manner of the leavers, $29 \%$ were planning to leave the hospital or the country, while only $(20 \%)$ of nurses who intended to leave, were planning to move to a non-hematology/oncology unit. Significantly, $<1 \%$ of the leavers, wanted to quit nursing and change their profession.

\section{Finding another job in nursing}

There was no significant relationship found between finding another job in nursing and intent to leave or stay $(\mathrm{P}>0.05)$ (Table 7$)$.

\begin{tabular}{|c|c|c|c|c|c|c|c|}
\hline & & \multicolumn{4}{|c|}{ Thinking about the next $1-3$ years } & \multirow[b]{2}{*}{ Test } & \multirow{3}{*}{ P-Value } \\
\hline & & \multicolumn{2}{|c|}{ Intend to stay } & \multicolumn{2}{|c|}{ Intend to leave } & & \\
\hline & & n & $\%$ & $\mathbf{n}$ & $\%$ & & \\
\hline \multirow[b]{2}{*}{ Gender } & Female & 96 & $50.80 \%$ & 93 & $49.20 \%$ & Chi-Square & 0.07 \\
\hline & Male & 23 & $67.60 \%$ & 11 & $32.40 \%$ & & \\
\hline \multirow[b]{4}{*}{ Age, y } & $<30$ & 43 & $55.80 \%$ & 34 & $44.20 \%$ & Chi-Square & 0.832 \\
\hline & $30-45$ & 58 & $52.70 \%$ & 52 & $47.30 \%$ & & \\
\hline & $46-55$ & 13 & $54.20 \%$ & 11 & $45.80 \%$ & & \\
\hline & $>55$ & 5 & $41.70 \%$ & 7 & $58.30 \%$ & Chi-Square & 0.027 \\
\hline \multirow[b]{2}{*}{ Marital Status } & Never Married & 41 & $44.60 \%$ & 51 & $55.40 \%$ & & \\
\hline & Ever Married & 78 & $59.50 \%$ & 53 & $40.50 \%$ & & \\
\hline \multirow[b]{4}{*}{ Educational degree } & Diploma & 31 & $59.60 \%$ & 21 & $40.40 \%$ & Cramer's V & 0.628 \\
\hline & Bachelor & 85 & $51.50 \%$ & 80 & & & \\
\hline & Master & 2 & $66.70 \%$ & 1 & $33.30 \%$ & Chi-Square & 0.583 \\
\hline & Other & 1 & $33.30 \%$ & 2 & $66.70 \%$ & & \\
\hline \multirow[b]{9}{*}{ Working unit } & B3 & 18 & $47.40 \%$ & 20 & $52.60 \%$ & & \\
\hline & CWA & 12 & $54.50 \%$ & 10 & $45.50 \%$ & & \\
\hline & CWB & 7 & $53.80 \%$ & 6 & $46.20 \%$ & & \\
\hline & CCC-OPD & 13 & $61.90 \%$ & 8 & $38.10 \%$ & & \\
\hline & Cancer Centre-OPD & 23 & $60.50 \%$ & 15 & $39.50 \%$ & & \\
\hline & $\mathrm{E} 1$ & 11 & $52.40 \%$ & 10 & $47.60 \%$ & & \\
\hline & E3 & 6 & $35.30 \%$ & 11 & $64.70 \%$ & & \\
\hline & $\mathrm{F} 2-2$ & 17 & $65.40 \%$ & 9 & $34.60 \%$ & & \\
\hline & F3 & 12 & $44.40 \%$ & 15 & $55.60 \%$ & & \\
\hline \multirow[b]{4}{*}{ Nationality } & Africa & 3 & $25.00 \%$ & 9 & $75.00 \%$ & Cramer's V & 0.014 \\
\hline & Arab & 32 & $62.70 \%$ & 19 & $37.30 \%$ & & \\
\hline & Asia & 71 & $58.70 \%$ & 50 & $41.30 \%$ & & \\
\hline & Australia & 1 & $14.30 \%$ & 6 & $85.70 \%$ & & \\
\hline
\end{tabular}




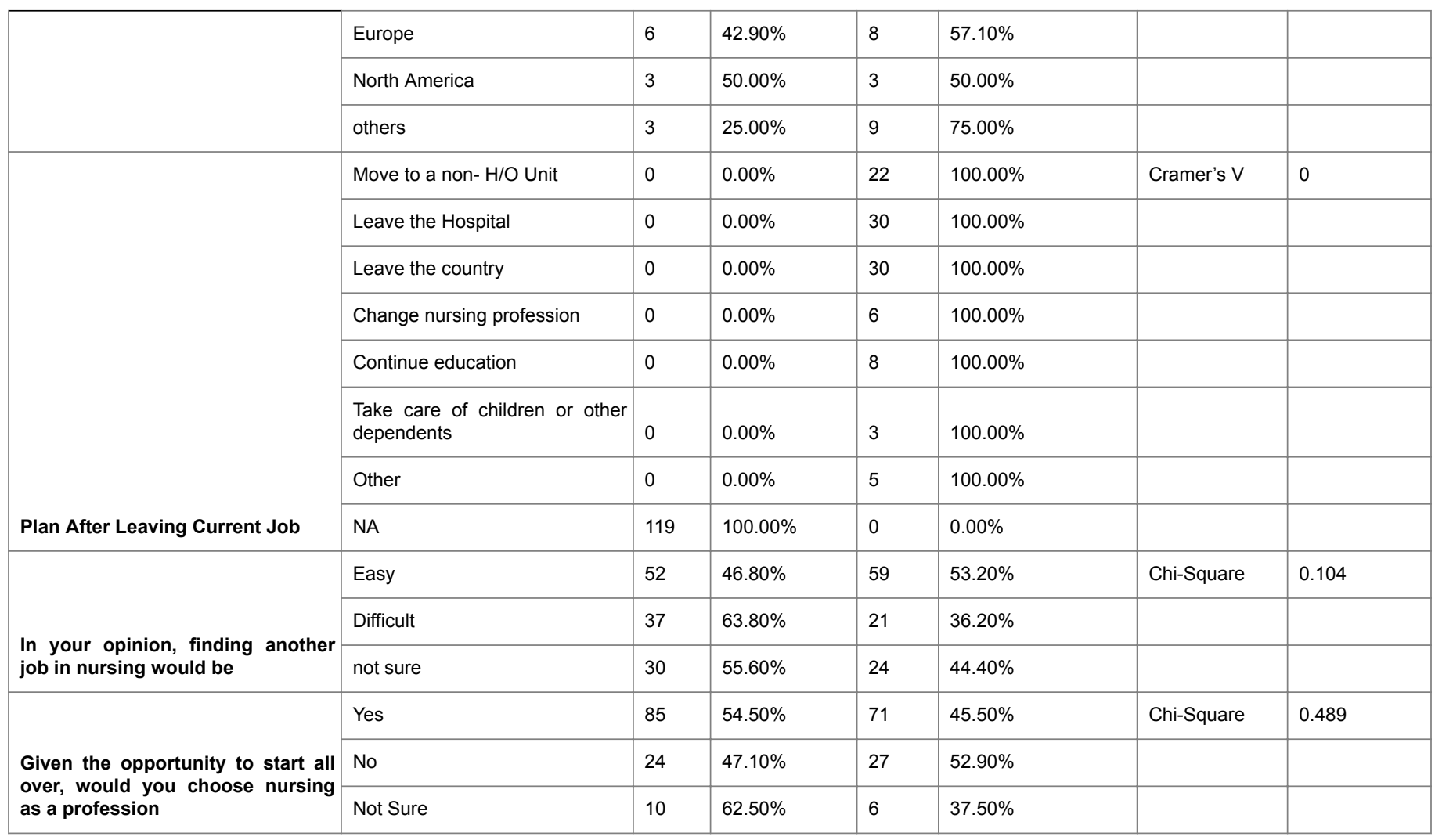

Table 7: Relationship between demographic characteristics and plan to leave or to stay.

Given the Opportunity to Choose Nursing as a Profession (45.5\%) of the leavers have answered yes and (53\%) have answered no for choosing nursing as a profession if they were given the chance again. Considering both those intending to leave and those intending to stay, more than $69 \%$ of the sample would have chosen nursing as profession if given the opportunity to start all over.

\section{Discussion}

The results of this study identify that near to half of the nurses from $\mathrm{H} / \mathrm{O}$ units intend to leave their current jobs within the coming 3 years. They are planning to leave their current unit, or totally leave the hospital. This alarming finding was consistent with the two study results of Sloane et al. [21] and Delobelle et al. [22] where half of the nurses included in both studies were planning to leave their jobs within two years and $30 \%$ of them, were planning to leave the country. It had been evident that nurses were leaving the $\mathrm{H} / \mathrm{O}$ area and the hospital, and this study has been able to give additional context, identifying some key demographics around who was leaving as well as addressing why staff is leaving.

Significantly, $21 \%$ of the leavers are thinking to leave the work at the $\mathrm{H} / \mathrm{O}$, and $8 \%$ are planning to leave the profession. This could be better explained as a result of staff become a nurse or working in $\mathrm{H} / \mathrm{O}$ nursing as a bridge to getting a different job outside nursing or $\mathrm{H} / \mathrm{O}$ with improved stress-free work conditions and better benefits $[3,4]$. The majority of the nurses working in the hospital where this study was conducted are from Asian and Arab countries. Many of these nurses may use the work in the KSA as a bridge to migrate to more developed countries for better life styles and better work conditions. Although, $35 \%$ of the sample said that they would easily found a new job in nursing, 33\% of them would not have chosen nursing as a profession, if they were given the chance to choose again. Moreover, $53.9 \%$ of the leavers would not have chosen nursing as profession if given the chance to choose from the beginning. This supports the conclusion that, $\mathrm{H} / \mathrm{O}$ nursing is a challenging job to choose as a profession or to sustain for a long time [4].

Language barriers could possibly affect the above findings. Arabic language is not the mother tongue for the vast majority of the expatriate nurses who work in the hospital. Moreover, those expatriate nurses are from different cultural backgrounds. Differences in languages and cultures may play a role and affect nurses' desire to stay and their perceptions toward their patients and their families. The expatriate nurses may develop a feeling of disrespect or can be misunderstood as nurses in a country of completely different language and culture [21]. Cultural competency was reported as having the strongest relationship with intention to leave among expatriate nurses working in the KSA [15]. It was found that expatriates who were originally from countries of similar cultures (i.e. from Arab countries) were less likely to intend to leave their work in the Kingdom [15]. The results of this study is in agreement that nationality has a significant effect on the nurses' decision to leave or stay in their current job ( $p$ value $=0.014$ ).

The best way of anticipating actual nurses' turnover or attrition is their intent to leave or stay and job satisfaction [3-5,21,23,25]. Results of previously mentioned studies were similar to the findings from this study which support that, nurses intend to leave when they are less 
satisfied, and intend to stay when they are more satisfied. Leaver nurses in this study were less satisfied in all of the eight satisfaction subscales as well as with the grand total job satisfaction (Table 2).

Furthermore, the grand job satisfaction was negatively associated with the intention to leave ( $\mathrm{r}=-0.179, \mathrm{P}$-value, 0.007) (Table 6). Indeed, these results support the findings of the previously mentioned studies $[3,5]$ and give a broader better explanation of how job satisfaction is one of the indicators to detect nurses' intention to leave. This result highlighted that being satisfied with different aspects of the job will encourage nurses to stay and decrease their intention to leave their current job in $\mathrm{H} / \mathrm{O}$ area, the hospital or even the nursing profession entirely. In brief, increased satisfaction reported by nurses, means a decreased intent to leave and decreased actual turnover.

The demographic characteristics were found to have a significant effect on the nurses' satisfaction and their plans to stay or to leave the job [25]. On the contrary, other study has found no correlation between job satisfaction and demographic characteristics [7].

When talking about job satisfaction, this study revealed significant different between gender and satisfaction with interaction opportunities ( $\mathrm{P}$ value $=0.003$ ). This could be an acknowledgement that female nurses are more empowered [23] as they are greater in number and more needed for work within the kingdom than male nurses. On the other hand, this study has found no significant differences between age groups in regard to nurses' intention to leave or stay ( $\mathrm{P}$ value $>0.05)$ although it was found that older nurses ( $>50$ years old) intended to leave more than any other younger age group (58\% of this group intended to leave). Younger nurses ( $<30$ years) intended to stay more than to leave. There was a strong significance between age and job satisfaction. Again, older nurses were less satisfied with co-workers than the younger ones (mean $=3$ and 4 , respectively, $\mathrm{P}$ value $=0.010$ ) which acknowledges that interaction with colleagues is very noteworthy to the older nurses to feel that they are still needed to stay in their job as expert resources and consultants to younger nurses. All age groups were equally satisfied with interaction opportunities ( mean $=3$ for all age groups, $P$ value $=0.020$ ).

In regard to marital status, never married nurses had more intent to leave than to stay in comparison to the ever married ones $(55.4 \%$ and $40.5 \%$ respectively, $\mathrm{P}$ value $=0.014$ ). These findings are similar to the findings of another study [3], when they found that $72 \%$ of the never married candidates intended to leave ( $P$ value $<0.001)$. The researcher in this study related these findings to that it is easier for the never married nurses to leave their current jobs and even the profession than those who were or still married as the formers have less responsibilities and commitments toward their dependents than the latter. The ever married nurses were less satisfied than the never married group with all satisfaction measures. A significant result was found when measuring the differences between marital status and satisfaction with balance between work and family (ever married mean $=2.33$ and never married mean $=2.64, \mathrm{P}$ value $=0.001$ ). From the writer's point of view, this was an expected finding, as ever married candidates have more family commitments and responsibilities than the never married candidates.

The study also showed no significant differences between the level education and the intention to leave ( $\mathrm{P}$ value $>0.05$ ). On the other hand, nurses with master degrees seemed to be more satisfied than the others (mean $=3.37$ for master holders and 3 for all other levels of education). Similarly [26] found that nurses with less than a Masters level of education intended to leave the profession more than those with a Masters level of education. However, the result of this study cannot be generalized to all nurses in different contexts as the number of the participants with a Masters level was low; less than $2 \%$ of the sample. On the contrary, Fitzpatrick et al. [23] found that significant difference in turnover intent was related to education level ( $\mathrm{P}$ value $<0.001$ ). Candidates with bachelor level of education were more likely to intend to leave than other candidates with other levels of education. These findings were related to the level of empowerment that nurses developed with different levels of education. The higher the education level, the more the empowerment, and could be more opportunities for different jobs and positions [23].

Since this study was conducted with expatriate nurses, it was significant that nationality affects their satisfaction. The least satisfied nurses were from Australia and Africa (Mean=2.87). Nurses from all nationalities except Europeans were least satisfied with balance between work and family. ( $\mathrm{P}$ value=0.002). This was related to expatriate experience, being away from the family in a foreign country [15] and may be due to the effect of a proportionately higher number of the ever married than the never married participants in addition to the differences in the culture, language and leadership styles.

All the participants from all $\mathrm{H} / \mathrm{O}$ units in this study were neutrally satisfied (mean $=3$ for each unit with grand job satisfaction). However, the differences between work unit and nurses' satisfaction with balance between work and family was significant $(P$ value $=0.000$ ). However, grand job satisfaction $(\mathrm{P}$ value $=0.020)$ was related to unit of work, which was associated with intent to leave. Correlation Coefficient (Pearson's) revealed that the strongest significant direct relationship was found between grand job satisfaction and nurses' perceived satisfaction with control and responsibility $(r=0.81, \mathrm{P}$-value $=0.000)$ (Table 5). The implication is that the unit being worked on may affect the intent to leave or turnover due to having a significant impact on the job satisfaction. The balance between work and family subscale measured nurses' satisfaction with control over what goes on at their work setting, opportunities for career advancement, amount of responsibilities, control on work conditions, and participation in decision-making. Nurses' plans to stay or leave were correlated to their control on work environment and availability of resources and equipment as the latter wasted their time and burned them out [27]. Multiple studies have shown correlation with control and responsibilities and job satisfaction, Mokoka et al. [28] found a strong correlation with job satisfaction and intent to leave and unfair opportunities to job development $[29,30]$ and noted a control over work included involvement in decision making and competency of work place and Takase et al. [31] found that nurses were more satisfied and intended to stay when they were involved in the decision making to establish policies and patient education. These findings are in congruence with the finding of this study and support the strong correlation between job satisfaction and satisfaction with control and responsibilities.

Significantly, it has been found in other studies that working at $\mathrm{H} / \mathrm{O}$ units increases the intent to leave current nursing job and burnout [32] due to work environmental conditions and different routes of exposure to chemo-therapeutic agents used for cancer treatment with its effects on the nurses' health [33]. The effect and stress caused by disease relapse and common sudden death of patients adds more burdens on the nurses working in such areas and influences their intent to leave $[34,35]$. Interestingly, it was found that all areas of specialties have an influence on nurses' rate of turnover [12,22]. In another study, with nurses who did not have a choice in place of work, their intent to leave 
was higher than those who have chosen their working unit [36]. It can be concluded from the results of this study and other related studies that working in $\mathrm{H} / \mathrm{O}$ area present significant challenges and stressors for the nurses working there and these are primary reasons behind their intention to leave.

\section{Conclusion and Recommendation}

This study has identified expatriate $\mathrm{H} / \mathrm{O}$ nurses perceived job satisfaction and how this perception supports predictions of staff intending to leave highlighting multiple factors that impact that perception. It may help the organization and nursing policy makers to focus their efforts to help nurses meet and achieve their needs, producing high levels of freedom and responsibility to develop their career, and to promote their retention. If managers are seeking to determine the potential for nurse's turnover, they need to be aware of the level of satisfaction with all aspects that measure and affect job satisfaction including work, managers, social and financial factors. This enables managers to focus on the main causes of turnover in order to correct these causes and to improve patient quality of care that delivered by nurses. Furthermore, this study alerts managers to the need for paying more attention to the differences in job satisfaction and intent to leave that nationalities cause, and there is a need to establish new strategies of recruitment, particularly looking at areas where it may be easy to recruit.

Managers need to find ways to improve the satisfaction of those nurses from nationalities with high level of turnover intention, in order to retain them for longer time to support the highest level of quality patient care. Nurses who were recruited from Asian nationalities and from Arab countries were more likely to stay than to leave, while nurses from Africa, Australia, Europe and North America, were more likely to leave. As intent to leave has been shown to be connected to nurses' satisfaction, it is important to both sustain the satisfaction of nurses but also seek to address specific needs that would improve the satisfaction of those nurses from countries with more leave intent, especially those nurses who come from countries with better lifestyles, higher opportunities to develop, more easy to find a job, and better educational backgrounds Reasons behind the low level of satisfaction among specific nationalities (i.e., Australia and Africa) could be explored further in order to compensate and make the required corrections.

Researchers have argued that the only most effective way for healthcare organizations to improve their performance is simply by increasing the staffing levels of their nurses. But such an approach can never work unless organizations are successful in finding effective solutions to reduce or manage nursing turnover and improve staff satisfaction level. For that, managers need to find strategies to improve nurses' job satisfaction. This can be achieved by conducting satisfaction surveys in a timely manner and use the result to proactively plan around any possible departure of nurses in order to decrease its impact on both the clients and the organization.

Links between job satisfaction, turnover intention and patient outcomes was not studied in this research. Research that directly explores this potential relationship is recommended. This study focused on the relationships between expatriate nurses' job satisfaction, their intent to leave, and other factors related to demographic characteristics and work environment in the H/O area. The writer suggests that future research should explore the influence of job satisfaction on intention to leave among bigger sample of $\mathrm{H} / \mathrm{O}$ expatriate nurses that covers all hospitals of the Kingdom of Saudi Arabia and compare the results to other expatriate nurses working in other clinical areas and link results of the suggested study to work conditions including work efforts and type of care provided.

\section{References}

1. Cowden T, Cummings G, Profetto-McGrath J (2011) Leadership practices and staff nurses' intent to stay: A systematic review. J Nurs Manag 19: 461-477.

2. Cowden TL, Cummings GG (2012) Nursing theory and concept development: a theoretical model of clinical nurses intentions to stay in their current positions. Journal of Advanced Nursing 68: 1646-1657.

3. El-Jardali F, Dimassi H, Dumit N, Jamal D, Mouro G (2009) A national cross-sectional study on nurses' intent to leave and job satisfaction in Lebanon: implications for policy and practice. BMC Nurs 8: 3 .

4. El-Jardali F, Merhi M, Jamal D, Dumit N, Mouro G (2009) Assessment of nurse retention challenges and strategies in Lebanese hospitals: The perspective of nursing directors. J Nurs Manag 17: 453-462.

5. Galletta M, Portoghese I, Battistelli A (2011) Intrinsic motivation, job autonomy and turnover intention in the italian healthcare: The mediating role of affective commitment. Journal of Management Research 3: 1-19.

6. Galletta M, Portoghese I, Penna MP, Battistelli A, Saiani L (2011) Turnover intention among Italian nurses: The moderating roles of supervisor support and organizational support. Nurs Health Sci 13: 184-191.

7. Gurková E, Cáp J, Žiaková K, Durišková M (2012) Job satisfaction and emotional subjective well-being among Slovak nurses. International Nursing Review 59: 94-100.

8. Liu C, Zhang L, Ye W, Zhu J, Cao J, et al. (2012) Job satisfaction and intention to leave: a questionnaire survey of hospital nurses in Shanghai of China. J Clin Nurs 21: 255-263.

9. Ravari A, Bazargan M, Vanaki Z, Mirzaei T (2012) Job satisfaction among Iranian hospital-based practicing nurses: Examining the influence of selfexpectation, social interaction and organisational situations. Journal of Nursing Management 20: 522-533.

10. Cummings GG, Olson K, Hayduk L, Bakker D, Fitch M, et al. (2008) The relationship between nursing leadership and nurses' job satisfaction in Canadian oncology work environments. J Nurs Manag 16: 508-518.

11. Chan EY, Morrison P (2000) Factors influencing the retention and turnover intentions of registered nurses in a Singapore hospital. Nursing and Health Science 2: 113-121.

12. Brewer CS, Kovner CT, Greene W, Tukov-Shuser M, Djukic M (2011) Predictors of actual turnover in a national sample of newly licensed registered nurses employed in hospitals. Journal of Advanced Nursing 68: 521-538.

13. Choi SP, Cheung K, Pang SM (2013) Attributes of nursing work environment as predictors of registered nurses' job satisfaction and intention to leave. J Nurs Manag 21: 429-439.

14. Newman K, Maylor U (2002) The nurse satisfaction, service quality and nurse retention chain: Implications for management of recruitment and retention. Journal of Health Organization and Management 16: 271-291.

15. Bozionelos N (2009) Expatriation outside the boundaries of the multinational corporation: A study with expatriate nurses in Saudi Arabia. Human Resource Management 48: 111-134.

16. Hunt ST (2009) Nursing turnover: Costs, causes, solutions. Success Factors.

17. Bae S (2008) Nursing unit turnover, workgroup processes and unit-level patient outcomes. The University of North Carolina at Chapel Hill.

18. Bae SH, Mark B, Fried B (2010) Impact of nursing unit turnover on patient outcomes in hospitals. J Nurs Scholarsh 42: 40-49.

19. Abu-Zinada S (2004) The situation of Saudi nursing. Health Forum 52: 42-43.

20. Almalki M, FitzGerald G, Clark M (2011) The nursing profession in Saudi Arabia: An overview. Int Nurs Rev 58: 304-311. 
Citation: Dagamseh MS, Haddad A (2017) Nurses Intent to Leave and Job Satisfaction in Hematology/Oncology Areas: Implications for Policy and Practice. J Nurs Care 5: 363. doi:10.4172/2167-1168.1000363

Page 15 of 15

21. Sloane PD, Williams CS, Zimmerman S (2010) Immigrant status and intention to leave of nursing assistants in U.S. nursing homes. J Am Geriatr Soc 58: 731-737.

22. Delobelle P, Rawlinson JL, Ntuli S, Malatsi I, Decock R, et al. (2011) Job satisfaction and turnover intent of primary healthcare nurses in rura South Africa: A questionnaire survey. Journal of Advanced Nursing 67: 371-383.

23. Fitzpatrick JJ, Campo TM, Graham G, Lavandero R (2010) Certification, empowerment and intent to leave current position and the profession among critical care nurses. Am J Crit Care 19: 218-226.

24. Tsai Y, Wu SW (2010) The relationships between organisational citizenship behaviour, job satisfaction and turnover intention. Journal of Clinical Nursing 19: 3564-3574.

25. De Milt DG, Fitzpatrick JJ, Sister Rita McNulty (2011) Nurse practitioners' job satisfaction and intent to leave current positions, the nursing profession and the nurse practitioner role as a direct care provider. J Am Acad Nurse Pract 23: 42-50.

26. Borkowski N, Amann R, Song S, Weiss C (2007) Nurses intent to leave the profession: Issues related to gender, ethnicity and educational level. Health Care Management Review 3: 160-167.

27. Tourangeau AE, Cummings G, Cranley LA, Ferron EM, Harvey S (2010) Determinants of hospital nurse intention to remain employed Broadening our understanding. J Adv Nurs 66: 22-32.

28. Mokoka E, Oosthuizen MJ, Ehlers VJ (2010) Retaining professional nurses in South Africa: Nurse managers perspectives. Health SA Gesondheid 15: 103-111.
29. Pearson A, Pallas L, Thomson D, Doucette E, Tucker D, et al. (2006) Systematic review of evidence on the impact of nursing workload and staffing on establishing healthy work environments. Int J Evid Based Healthc 4: 337-384.

30. Cho SH, Lee JY, Mark BA, Yun SC (2012) Turnover of new graduate nurses in their first job using survival analysis. J Nurs Scholarsh 44: 63-70.

31. Takase M, Maude P, Manias E (2006) The impact of role discrepancy on nurses intention to quit their jobs. J Clin Nurs 15: 1071-80.

32. Liakopoulou M, Panaretaki I, Papadakis V, Katsika A, Sarafidou J, et al. (2008) Burnout, staff support and coping in Pediatric Oncology. Support Care Cancer 16: 143-150.

33. Kopp B, Schierl R, Nowak D (2013) Evaluation of working practices and surface contamination with antineoplastic drugs in outpatient oncology health care settings. Int Arch Occup Environ Health 86: 47-55.

34. Hinds PS, Puckett P, Donohoe M, Milligan M, Payne K, et al. (1994) The impact of a grief workshop for pediatric oncology nurses on their grief and perceived stress. J Pediatr Nurs 9: 388-397.

35. Hinds PS, Sanders CB, Srivastava DK, Hickey S, Jayawardene D, et al. (1998) Testing the stress-response sequence model in paediatric oncology nursing. Journal Of Advanced Nursing 28: 1146-1157.

36. Beecroft PC, Dorey F, Wenten M (2008) Turnover intention in new graduate nurses: A multivariate analysis. J Adv Nurs 62: 41-52. 